\title{
Status of Multi-Geared Small Scale Fishery Operated in Koggala Lagoon and Adjacent Coastal Area
}

\author{
Hansika J.G.C. and De Croos M.D.S.T.* \\ Department of Aquaculture and Fisheries, Wayamba University of Sri Lanka, Sri Lanka \\ *dileepa_dc@yahoo.com
}

\begin{abstract}
Management of multi-species and multi-geared fisheries is challenging due to conflicts and competitions of fishers. However, such a complex fishery exists for the last 100 years in Koggala lagoon, which extends only for 727 ha area, in southern Sri Lanka. This study evaluated the fish catch and income sharing mechanisms which enable to survive these fisheries in highly dynamic biological and socio-economical environments. Eight landing sites at the lagoon body, lagoon mouth and adjacent coast were weekly visited from April to August 2015 in drawing random samples to determine gear-based catch per unit effort (CPUE), species diversity and income levels. Questionnaires and discussions among fishers were used to identify potentials and threats for the fishery. Dominant 25 fish species and 6 shrimp species were observed in the catches of 11 fishing methods including snares, a fish trap which was not recorded from Koggala lagoon. During the study period high variations of CPUE were observed within and among fishing methods. Hierarchical cluster analysis based on presentabsent data showed two clearly separated clusters $(\mathrm{P}<0.05)$. Though the educational level and years of experience significantly differed between lagoon and coastal fishers $(\mathrm{P}<0.05)$, the respective income was not significantly different. Targeting different species and different life history stages by different types of gear seems to be the key in sustaining multi-geared fisheries in the lagoon for last 100 years. Though school education does not have any effect on selecting fishing location, fishing experience seems to be important in operating some specific gear. Species aggregation and their susceptibility for different gear reveled in this study must be important in developing fishery management strategies for the lagoon. Despite to the effective catch sharing mechanism, the sand bar formation, which is the most concerned issue by both lagoon and coastal fishers, will determine whether Koggala lagoon fisheries will survived for another 100 years due to its effects on altering species diversity.
\end{abstract}

Keywords: Artisanal fisheries, Catch composition, Socio-economic status

Proceedings of the International Forestry and Environment Symposium 2016, Department of Forestry and Environmental Science, University of Sri Jayewardenepura, Sri Lanka. 